\title{
Association between the Thigh Muscle and Insulin Resistance According to Body Mass Index in Middle- Aged Korean Adults
}

\author{
Ji Eun Heo ${ }^{1,2}$, Jee-Seon Shim ${ }^{2,3}$, Hokyou Lee ${ }^{3}$, Hyeon Chang Kim ${ }^{2,3}$ \\ ${ }^{1}$ Department of Public Health, Yonsei University Graduate School, Seoul, \\ ${ }^{2}$ Cardiovascular and Metabolic Disease Etiology Research Center, ${ }^{3}$ Department of Preventive Medicine, Yonsei University College of Medicine, Seoul, Korea
}

Background: We examined the associations between thigh muscle area (TMA) and insulin resistance (IR) according to body mass index (BMI) in middle-aged Korean general population.

Methods: TMA was measured using quantitative computed tomography and corrected by body weight (TMA/Wt) in 1,263 men, 788 premenopausal women, and 1,476 postmenopausal women all aged 30 to 64 years. The tertiles of TMA/Wt were calculated separately for men and for premenopausal and postmenopausal women. Homeostatic model assessment for insulin resistance (HOMA-IR) was performed using fasting blood glucose and insulin levels, and increased IR was defined according to sex-specific, top quartiles of HOMA-IR. Associations between the TMA/Wt tertiles and increased IR according to the BMI categories $(<25$ and $\geq 25 \mathrm{~kg} / \mathrm{m}^{2}$ ) were assessed using multivariable logistic regression analysis.

Results: In men with higher BMIs, but not in those with lower BMIs, the presence of an increased IR had significantly higher odds ratios in the lower TMA/Wt tertiles, even after adjustment for visceral fat area. However, in premenopausal and postmenopausal women, there was no significant inverse association between TMA/Wt tertiles and increased IR, regardless of BMI category.

Conclusion: Our findings suggest that the thigh muscle is inversely associated with IR in men, particularly in those with higher BMIs.

Keywords: Body composition; Insulin resistance; Muscles; Sarcopenia; Tomography, X-ray computed

\section{INTRODUCTION}

Sarcopenia, the degenerative loss of skeletal muscle mass, could be associated with a cardio-metabolic risk, metabolic disease, and psychological disorders [1-3]. Many studies have reported a significant inverse association between muscle mass and insulin resistance (IR). However, most of these studies were conducted in older adults or people with chronic conditions, such as diabetes mellitus, chronic obstructive pulmonary disease, or end-stage renal disease [4-8]. Some studies have been conducted in general populations [2,4,8-11], but only few of them targeted middle-aged population [2,11]. And the results were inconsistent. One study found a significant association between low muscle mass and higher IR in a middle-aged population [11]. Another noted a significant association between low muscle mass and higher IR in an older adult population, but was unable to document the same association in a middle-aged population [2]. Although the initiation of muscle decline starts in the middle age, little information is available regarding the independent impact of low muscle mass and IR in the middle-aged general population.

Several lifestyle factors, including smoking, alcohol con-
Corresponding author: Hyeon Chang Kim (D) https://orcid.org/0000-0001-7867-1240 Department of Preventive Medicine, Yonsei University College of Medicine, 50 Yonseiro, Seodaemun-gu, Seoul 03722, Korea

E-mail: hckim@yuhs.ac

Received: Jun. 10, 2019; Accepted: Oct. 7, 2019
This is an Open Access article distributed under the terms of the Creative Commons Attribution Non-Commercial License (https://creativecommons.org/licenses/by-nc/4.0/) which permits unrestricted non-commercial use, distribution, and reproduction in any medium, provided the original work is properly cited. 
sumption, exercise, and protein intake, could be determinants of decreases in muscle mass and function, and age-related muscle decline could be more prominent in men than in women [12]. Although many characteristics differ between men and women, most studies on associations between muscle mass and IR have only addressed these differences in their analyses by adjustment, instead of stratification. Also, previous studies have demonstrated different trends in muscle and obesity indicators, including visceral fat, according to the menopausal status of women [13]. These differences according to sex and menopausal status could modify the relationships between muscle mass and IR, but there are not enough studies and the mechanisms are not well established. In addition, it should be investigated whether the impacts of the muscle mass on IR are independent from those of known risk factors, including lifestyle factors and obesity status [14-16].

Accordingly, we sought to evaluate the independent relationship between thigh muscle area (TMA) measured using quantitative computed tomography (QCT) and IR according to different body mass index (BMI) categories in middle-aged, relatively healthy, men and premenopausal and postmenopausal women.

\section{METHODS}

\section{Study population}

The present study utilized data from the Cardiovascular and Metabolic Disease Etiology Research Center (CMERC) cohort study, which was designed to recruit members of the general population residing in four districts (Seoul, Goyang, Gimpo, and Incheon) in South Korea. The inclusion criteria were 30 to 64 years of age, residing more than 8 months of the year at current residence with no plans to move over the next 2 years, and having the ability to provide verbal or written consent to participate in the study. Participants were excluded if they had been diagnosed with cancer within the last 2 years or were currently being treated for cancer; had a history of myocardial infarction, stroke, or heart failure; were currently involved in pharmaceutical trials; or were currently pregnant or reported the possibility of being pregnant on the day of registration. The detailed methods for the CMERC cohort study have been reported elsewhere [17]. This study initially enrolled 4,060 participants between 2013 and 2017. All participants completed health questionnaires and examinations according to a predefined protocol. Those with missing TMA variables $(n=268)$, those who were treated for diabetes mellitus $(n=190)$, and those with a BMI under $18.5 \mathrm{~kg} / \mathrm{m}^{2}(n=75)$ were excluded from the current analysis. Finally, a cross-sectional analysis was conducted of the remaining 1,263 men, 788 premenopausal women, and 1,476 postmenopausal women. All participants provided written informed consent, and the study protocol was approved by the Institutional Review Board of Severance Hospital at Yonsei University College of Medicine (IRB No. 4-2013-0661).

\section{Measurements}

All participants were individually interviewed using standardized questionnaires to obtain information regarding demographics, health behaviors, and medication use. Trained interviewers conducted face-to-face interviews and administered questionnaires according to a standard protocol. Health behaviors included smoking status (current smoker, former smoker, or non-smoker), alcohol intake (current heavy drinker, current non-heavy drinker, former drinker, or non-drinker), physical activity, and sleep duration. Average alcohol consumption (in grams per drinking day) was calculated using the average number of alcoholic beverages consumed and the frequency of alcohol consumption, and then converted into the amount of ethanol in grams. Current heavy drinking was defined as drinking $>40 \mathrm{~g}$ ethanol per drinking day for men and $>20$ g per drinking day for women for two or more times a week [18]. Physical activity was assessed using the International Physical Activity Questionnaire-Short Form. For this study, high activity referred to activities that considerably increased an individual's respiratory effort, whereas moderate activity referred to those that moderately increased respiratory effort. Regular exercise was defined as moderate-to-high-intensity physical activity performed at least three times per week. Sleep duration was recorded in hours per day (hr/day) and as the average over the past year. Medication use (antihypertensive and lipid-lowering) was assessed by self-reports.

The study participants wore lightweight clothing for convenient and reliable examinations. BMI was calculated as the body weight divided by the standing height squared $\left(\mathrm{kg} / \mathrm{m}^{2}\right)$. Waist circumference was assessed to the nearest $0.1 \mathrm{~cm}$ at the midpoint between the lower border of the rib cage and the iliac crest using an ergonomic circumference-measuring tape (SECA 201; SECA, Hamburg, Germany). Participants rested for 5 minutes before the blood pressure measurement, and systolic and diastolic blood pressures were repeatedly measured 
three times at 2-minute intervals. The average of the second and third measurements was used for analysis. Hypertension was defined as a systolic blood pressure $\geq 140 \mathrm{~mm} \mathrm{Hg}$, diastolic blood pressure $\geq 90 \mathrm{~mm} \mathrm{Hg}$, or a self-report of current use of an antihypertensive medication.

Blood samples were collected from the antecubital vein after the patients had fasted for at least 8 hours. Fasting blood glucose concentrations were measured using a colorimetry method (ADVIA 1800 Auto Analyzer; Siemens Medical Solutions, Malvern, PA, USA). Serum insulin concentrations were measured with a radioimmunoassay (SR-300; Stratec, Birkenfeld, Germany). Glycosylated hemoglobin concentrations were assessed using high-performance liquid chromatography (Variant II TURBO; Bio-Rad, Berkeley, CA, USA) according to the National Glycohemoglobin Standardization Program guidelines. IR was evaluated using the homeostasis model assessment of IR (HOMA-IR) and was calculated as follows: fasting plasma glucose $(\mathrm{mg} / \mathrm{dL}) \times$ fasting plasma insulin $(\mathrm{mIU} / \mathrm{mL}) /$ 405 [19]. Increased IR was defined as ranking in the top 25th percentile of HOMA-IR values separately for men $(\geq 2.711)$, premenopausal women $(\geq 2.148)$, and postmenopausal women $(\geq 2.318)$ [20]. Pre-diabetes was defined as a fasting glucose level of 100 to $126 \mathrm{mg} / \mathrm{dL}$ or a glycosylated hemoglobin level of 5.7 to $6.5 \%$ according to the American Diabetes Association criteria [21]. Total cholesterol, high-density lipoprotein cholesterol, low-density lipoprotein cholesterol, and triglyceride levels were measured using enzymatic methods (ADVIA 1800 Auto Analyzer). Dyslipidemia was defined as a total cholesterol level $\geq 240 \mathrm{mg} / \mathrm{dL}$, or a high-density lipoprotein cholesterol level $\leq 40 \mathrm{mg} / \mathrm{dL}$, or a low-density lipoprotein cholesterol level $\geq 160 \mathrm{mg} / \mathrm{dL}$, or a triglycerides level $\geq 200 \mathrm{mg} / \mathrm{dL}$ according to the Expert Panel on Detection, Evaluation, and Treatment of High Blood Cholesterol in Adults or as people who were taking lipid-lowering medication. C-reactive protein concentrations were determined with a turbidimetric immunoassay (ADVIA 1800 Auto Analyzer).

TMA and visceral fat area were measured by QCT using a Somatom Definition AS+ 128-channel CT (Siemens Healthcare, Forchheim, Germany), a Somatom sensation 64-channel CT (Siemens Healthcare), or a GE Lightspeed VCT scanner (General Electric Medical System, Milwaukee, WI, USA). Scanning was performed at $120 \mathrm{kVp}$ and $150 \mathrm{mAs}$ and a pitch of 1.0. Images were reconstructed with a 3-mm slice thickness. Voxels were separated into fat and muscle tissue according to Hounsfield units (HU): -190 to $-30 \mathrm{HU}$ for fat and 30 to 100
$\mathrm{HU}$ for muscle. All scanned data were analyzed using Aquarius intuition Viewer version 4.4.12 software (Terarecon, Foster City, CA, USA). TMA was recorded as the mean of right and left TMA values. TMA was found to be correlated with body size ( $r=0.566$ in men, $r=0.491$ in premenopausal women, and $r=0.463$ in postmenopausal women), indicating that participants with a larger body size may have larger TMA. Therefore, TMA was corrected by body weight (TMA/Wt, $\mathrm{cm}^{2} / \mathrm{kg}$ ) [22]. The participants were divided into three groups according to sex and menopausal-specific TMA/Wt tertiles: $<338.9$, 338.9377.7 , and $\geq 377.8$ for men; $<276.5,276.5-309.1$, and $\geq 309.2$ for premenopausal women; and <261.0, 261.0-292.4, and $\geq 292.5$ for postmenopausal women.

\section{Statistical analysis}

We evaluated differences in demographic characteristics among the three groups based on the TMA/Wt tertiles, and we used one-way analysis of variance for normally distributed variables, Kruskal-Wallis test for skewed variables, and chisquare test for categorical variables. The $P$ for trend was calculated using a contrast to test for linear trends in continuous variables and the Cochran-Armitage test for categorical variables.

Multivariable logistic regression analyses were used to assess independent associations between TMA/Wt (using tertiles and continuous variables) and the likelihood of an increased IR in three adjusted models: [1] adjusted for age; [2] additionally adjusted for systolic blood pressure, triglyceride level, smoking, alcohol intake, exercise, and sleep duration; and [3] additionally adjusted for visceral fat area. We conducted the above-mentioned analyses separately for men, premenopausal, and postmenopausal women, because there were significant interactions between sex and TMA/Wt $(P$ for interaction $=0.028)$ and a borderline interaction between menopausal status and TMA/Wt $(P$ for interaction $=0.065)$ in the presence of an increased IR and because both TMA/Wt and HOMA-IR significantly differed according to sex and menopausal status in women (Supplementary Table 1).

The associations between TMA/Wt (using tertiles and continuous variables) and the likelihood of an increased IR were also assessed in people with lower $\left(<25.0 \mathrm{~kg} / \mathrm{m}^{2}\right)$ and higher BMIs $\left(\geq 25.0 \mathrm{~kg} / \mathrm{m}^{2}\right)$ using the same models. Then, we also analyzed interactions between TMA/Wt tertiles and BMI categories for the presence of an increased IR in men and in premenopausal and postmenopausal women. All statistical tests 
were performed using SAS version 9.4 (SAS Institute, Cary, NC, USA). Statistical significance was defined as a two-sided $P$ value $<0.05$.

\section{RESULTS}

The general characteristics of the study participants are presented in Supplementary Table 1. This study included 1,263 men, 788 premenopausal women, and 1,476 postmenopausal women, with mean ages of 49.8, 43.0, and 57.1 years, respectively. Both mean TMA/Wt and median HOMA-IR values were significantly higher in men than in women. Mean TMA/ Wt was significantly higher in premenopausal than in postmenopausal women. Conversely, the median HOMA-IR was significantly higher in postmenopausal than in premenopausal women.

Table 1 shows the general characteristics of the men according to TMA/Wt tertiles. Men in the lower TMA/Wt tertiles tended to have higher obesity indices, blood pressure, triglycerides, and HOMA-IR values; higher frequencies of former smokers; and higher frequencies of hypertension, pre-diabetes mellitus, and dyslipidemia. Tables 2 and 3 show the general characteristics of the premenopausal and postmenopausal women, respectively, according to TMA/Wt tertiles. Both premenopausal and postmenopausal women in the lower TMA/ Wt tertiles tended to have higher obesity indices, blood pressure, lipid profile, HOMA-IR, C-reactive, and protein levels; higher frequencies of hypertension; and lower frequencies of regular exercise. Postmenopausal women in the lower TMA/ Wt tertiles tended to have higher frequencies of pre-diabetes mellitus and dyslipidemia and lower frequencies of current heavy drinking, unlike premenopausal women.

Table 4 presents the results of the multivariable logistic regression models for the associations between TMA/Wt (as tertiles and continuous variables) and increased IR in men, premenopausal, and postmenopausal women. In men, even after adjustment for potential confounders, including visceral fat area, the presence of an increased IR was significantly higher in the lower TMA/Wt tertile (odds ratio [OR], 1.63; 95\% confidence interval [CI], 1.10 to 2.45 ), but was not significant in the middle tertile (OR, $1.05 ; 95 \% \mathrm{CI}, 0.70$ to 1.58 ), relative to the upper tertile. In this model, which included TMA/Wt as a continuous variable, the multivariable-adjusted OR increased by 1.06 (95\% CI, 1.02 to 1.10 ) per $10 \mathrm{~cm}^{2} / \mathrm{kg}$ lower TMA/Wt. However, in both premenopausal and postmenopausal wom- en, an association between TMA/Wt and increased IR was not observed after additionally adjustment for visceral fat area. We also repeated the analysis using fasting glucose and fasting insulin levels as dependent variables instead of HOMA-IR and observed similar results (data not shown).

Table 5 presents the associations between TMA/Wt and increased IR according to the BMI categories in men. Among men with lower BMIs, the presence of an increased IR had higher, but non-significant, ORs in the lower and middle TMA/Wt tertiles. However, among men with higher BMIs, the presence of an increased IR showed significantly higher ORs in the lower TMA/Wt tertiles than in the upper TMA/Wt tertile. There was a significant interaction between the TMA/Wt tertiles and BMI categories in the presence of an increased IR in men ( $P$ for interaction $=0.046$ ).

Conversely, there were no significant associations between TMA/Wt and increased IR, regardless of BMI category, in premenopausal and postmenopausal women. Also, there were no significant interactions between TMA/Wt tertiles and BMI categories in the presence of an increased IR in premenopausal ( $P$ for interaction $=0.093)$ or postmenopausal $(P$ for interaction $=0.853$ ) women (Supplementary Table 2$)$.

\section{DISCUSSION}

The current study found that a lower thigh muscle was independently associated with a higher IR, even after adjustment for traditional risk factors, in a cohort of middle-aged Korean men with higher BMIs, but not in men with lower BMIs. This trend was not observed in premenopausal and postmenopausal women, regardless of the BMI category.

Associations between muscle mass and IR have been reported in animal [23] and human studies [1,2,11,24-27], including a gene analysis [28]. One of these results is consistent [24] with ours, and one is inconsistent [25]. Some studies are incomparable $[2,11,26,27]$ because they did not stratify by the obesity status. One study [24] reported that people with a lower muscle mass and higher BMIs had significantly higher ORs for IR, particularly in a middle-aged population. Another study [25] suggested that people with a lower muscle mass and lower BMIs had a significantly higher OR for IR. This inconsistent results might be due to not adjusting for central obesity, which could be highly correlated with the IR, particularly in the lower-BMI population [29]. Other studies [2,11,26,27] had similar results with ours; however, comparison is difficult because a 
Table 1. Characteristics in men $(n=1,263)$ according to TMA/Wt tertiles

\begin{tabular}{|c|c|c|c|c|c|}
\hline \multirow{2}{*}{ Variable } & \multicolumn{3}{|c|}{ TMA/Wt tertiles } & \multirow{2}{*}{$\begin{array}{c}P \text { for } \\
\text { difference }\end{array}$} & \multirow{2}{*}{$\begin{array}{l}P \text { for } \\
\text { trend }\end{array}$} \\
\hline & Lower $\left(<338.9 \mathrm{~cm}^{2} / \mathrm{kg}\right)$ & Middle $\left(338.9-377.7 \mathrm{~cm}^{2} / \mathrm{kg}\right)$ & Upper $\left(\geq 377.8 \mathrm{~cm}^{2} / \mathrm{kg}\right)$ & & \\
\hline Age, yr & $50.3 \pm 10.6$ & $50.7 \pm 10.1$ & $48.3 \pm 10.1$ & 0.002 & 0.167 \\
\hline $\mathrm{BMI}, \mathrm{kg} / \mathrm{m}^{2}$ & $26.3 \pm 3.2$ & $24.6 \pm 2.4$ & $23.8 \pm 2.3$ & $<0.001$ & $<0.001$ \\
\hline Waist circumference, $\mathrm{cm}$ & $91.6 \pm 8.0$ & $86.6 \pm 5.9$ & $82.3 \pm 6.3$ & $<0.001$ & $<0.001$ \\
\hline Visceral fat area, $\mathrm{cm}^{2}$ & $143.6 \pm 48.9$ & $118.7 \pm 40.8$ & $88.9 \pm 36.3$ & $<0.001$ & $<0.001$ \\
\hline SBP, mm Hg & $128.0 \pm 13.5$ & $124.7 \pm 13.7$ & $123.0 \pm 13.2$ & $<0.001$ & $<0.001$ \\
\hline DBP, mm Hg & $83.1 \pm 10.2$ & $80.5 \pm 9.7$ & $79.2 \pm 9.7$ & $<0.001$ & $<0.001$ \\
\hline Total cholesterol, mg/dL & $197.1 \pm 35.2$ & $199.9 \pm 35.8$ & $196.7 \pm 30.9$ & 0.333 & 0.597 \\
\hline HDL-C, mg/dL & $48.9 \pm 12.2$ & $50.7 \pm 11.7$ & $54.4 \pm 12.8$ & $<0.001$ & $<0.001$ \\
\hline LDL-C, mg/dL & $122.3 \pm 32.8$ & $123.9 \pm 34.3$ & $120.9 \pm 28.1$ & 0.427 & 0.938 \\
\hline Triglycerides, mg/dL & $141.0(104.0-209.0)$ & $136.0(97.0-194.0)$ & $117.0(80.0-163.0)$ & $<0.001$ & $<0.001$ \\
\hline Fasting glucose, mg/dL & $92.0(86.0-102.0)$ & $91.0(84.0-99.0)$ & $89.0(83.0-94.0)$ & $<0.001$ & $<0.001$ \\
\hline Fasting insulin, uIU/mL & $10.3(7.8-13.7)$ & $8.7(6.8-10.9)$ & $7.5(6.0-9.8)$ & $<0.001$ & $<0.001$ \\
\hline $\mathrm{HbA1c}, \%$ & $5.6(5.4-5.9)$ & $5.5(5.3-5.8)$ & $5.4(5.2-5.6)$ & $<0.001$ & $<0.001$ \\
\hline HOMA-IR & $2.39(1.74-3.35)$ & $2.00(1.52-2.58)$ & $1.64(1.30-2.20)$ & $<0.001$ & $<0.001$ \\
\hline Increased IR, \% & $170(40.4)$ & $92(21.9)$ & $54(12.8)$ & $<0.001$ & $<0.001$ \\
\hline $\mathrm{C}$-reactive protein, $\mathrm{mg} / \mathrm{L}$ & $0.9(0.5-1.9)$ & $0.7(0.4-1.3)$ & $0.5(0.3-1.1)$ & 0.068 & 0.163 \\
\hline Hypertension & $188(44.7)$ & $132(31.4)$ & $110(26.1)$ & $<0.001$ & $<0.001$ \\
\hline Pre-diabetes mellitus & $181(43.0)$ & $166(39.4)$ & $111(26.4)$ & $<0.001$ & $<0.001$ \\
\hline Dyslipidemia & $232(55.1)$ & $222(52.7)$ & $155(36.8)$ & $<0.001$ & $<0.001$ \\
\hline Antihypertensive medication & $97(23.0)$ & $66(15.7)$ & $42(10.0)$ & $<0.001$ & $<0.001$ \\
\hline Lipid-lowering medication & $40(9.5)$ & $36(8.6)$ & $23(5.5)$ & 0.075 & 0.029 \\
\hline Smoking status & & & & 0.042 & 0.827 \\
\hline Non-smoker & $93(22.1)$ & $84(19.9)$ & $115(27.3)$ & & \\
\hline Former smoker & $197(46.8)$ & $180(42.8)$ & $172(40.9)$ & & \\
\hline Current smoker & $131(31.1)$ & $157(37.3)$ & $134(31.8)$ & & \\
\hline Alcohol intake & & & & 0.409 & 0.231 \\
\hline Non-drinker & $35(8.3)$ & $24(5.7)$ & $40(9.5)$ & & \\
\hline Former drinker & $21(5.0)$ & $25(5.9)$ & $27(6.4)$ & & \\
\hline Current non-heavy drinker & $200(47.5)$ & $208(49.4)$ & $186(44.2)$ & & \\
\hline Current heavy drinker & $165(39.2)$ & $164(39.0)$ & $168(39.9)$ & & \\
\hline Regular exercise & $270(64.1)$ & $267(63.4)$ & $295(70.1)$ & 0.082 & 0.063 \\
\hline Sleep duration, hr/day & $6.9 \pm 1.2$ & $6.8 \pm 1.2$ & $7.0 \pm 1.1$ & 0.096 & 0.601 \\
\hline
\end{tabular}

Values are presented as mean \pm standard deviation, median (interquartile range), or number (\%). $P$ values were derived using the independent $t$ test, Wilcoxon rank sum test, or chi-square test.

TMA, thigh muscle area; Wt, weight; BMI, body mass index; SBP, systolic blood pressure; DBP, diastolic blood pressure; HDL-C, high-density lipoprotein cholesterol; LDL-C, low-density lipoprotein cholesterol; HbAlc, glycosylated hemoglobin; HOMA-IR, homeostasis model assessment of insulin resistance; IR, insulin resistance.

stratified analysis according to sex and obesity status was not performed in those studies. Although two of them [26,27] conducted a longitudinal analysis between low muscle mass and higher IR it might be hard to identify an independent effect of the low muscle because it was not adjusted by the body size. Except for one study [26], most previous studies used an 
Table 2. Characteristics in premenopausal women $(n=788)$ according to TMA/Wt tertiles

\begin{tabular}{|c|c|c|c|c|c|}
\hline \multirow{2}{*}{ Variable } & \multicolumn{3}{|c|}{ TMA/Wt tertiles } & \multirow{2}{*}{$\begin{array}{c}P \text { for } \\
\text { difference }\end{array}$} & \multirow{2}{*}{$\begin{array}{l}P \text { for } \\
\text { trend }\end{array}$} \\
\hline & Lower $\left(<276.5 \mathrm{~cm}^{2} / \mathrm{kg}\right)$ & Middle $\left(276.5-309.1 \mathrm{~cm}^{2} / \mathrm{kg}\right)$ & Upper $\left(\geq 309.2 \mathrm{~cm}^{2} / \mathrm{kg}\right)$ & & \\
\hline Age, yr & $43.6 \pm 6.9$ & $43.3 \pm 6.9$ & $42.0 \pm 7.0$ & 0.019 & 0.056 \\
\hline BMI, kg/m² & $24.4 \pm 3.2$ & $22.9 \pm 2.6$ & $21.6 \pm 2.0$ & $<0.001$ & $<0.001$ \\
\hline Waist circumference, $\mathrm{cm}$ & $80.3 \pm 8.1$ & $76.3 \pm 6.3$ & $72.3 \pm 5.9$ & $<0.001$ & $<0.001$ \\
\hline Visceral fat area, $\mathrm{cm}^{2}$ & $84.20 \pm 32.70$ & $75.80 \pm 30.70$ & $58.10 \pm 25.6$ & $<0.001$ & $<0.001$ \\
\hline SBP, mm Hg & $113.5 \pm 12.7$ & $110.7 \pm 12.6$ & $108.6 \pm 11.7$ & $<0.001$ & 0.001 \\
\hline DBP, $\mathrm{mm} \mathrm{Hg}$ & $73.8 \pm 9.5$ & $72.2 \pm 9.0$ & $71.1 \pm 8.5$ & 0.001 & 0.002 \\
\hline Total cholesterol, mg/dL & $195.6 \pm 32.2$ & $191.1 \pm 30.6$ & $188.9 \pm 33.8$ & 0.051 & 0.021 \\
\hline $\mathrm{HDL}-\mathrm{C}, \mathrm{mg} / \mathrm{dL}$ & $60.8 \pm 14.1$ & $61.4 \pm 13.9$ & $64.4 \pm 15.4$ & 0.011 & 0.054 \\
\hline LDL-C, mg/dL & $117.8 \pm 31.2$ & $113.7 \pm 27.1$ & $108.2 \pm 29.8$ & 0.002 & 0.004 \\
\hline Triglycerides, mg/dL & $93.5(73.0-123.0)$ & $85.0(65.0-110.0)$ & $79.0(59.0-107.0)$ & $<0.001$ & 0.004 \\
\hline Fasting glucose, mg/dL & $85.0(80.0-90.0)$ & $86.0(80.0-91.0)$ & $83.0(79.0-89.0)$ & 0.002 & 0.696 \\
\hline Fasting insulin, uIU/mL & $8.3(7.0-10.7)$ & $7.9(6.6-9.8)$ & $7.1(6.0-8.8)$ & $<0.001$ & 0.002 \\
\hline HbAlc, $\%$ & $5.4(5.2-5.6)$ & $5.4(5.2-5.6)$ & $5.4(5.2-5.5)$ & 0.622 & 0.691 \\
\hline HOMA-IR & $1.75(1.43-2.34)$ & $1.69(1.36-2.21)$ & $1.49(1.24-1.88)$ & $<0.001$ & 0.009 \\
\hline Increased IR, \% & $82(31.3)$ & $73(27.8)$ & $42(16.0)$ & $<0.001$ & $<0.001$ \\
\hline C-reactive protein, $\mathrm{mg} / \mathrm{L}$ & $0.6(0.4-1.3)$ & $0.5(0.3-1.1)$ & $0.3(0.2-0.6)$ & $<0.001$ & 0.021 \\
\hline Hypertension & $33(12.6)$ & $22(8.4)$ & $19(7.2)$ & 0.085 & 0.035 \\
\hline Pre-diabetes mellitus & $55(21.0)$ & $57(21.7)$ & $45(17.1)$ & 0.369 & 0.265 \\
\hline Dyslipidemia & $47(17.9)$ & $52(19.8)$ & $45(17.1)$ & 0.721 & 0.805 \\
\hline Antihypertensive medication & $14(5.3)$ & $6(2.3)$ & $8(3.0)$ & 0.143 & 0.155 \\
\hline Lipid-lowering medication & $3(1.2)$ & $7(2.7)$ & $6(2.3)$ & 0.439 & 0.357 \\
\hline Smoking status & & & & 0.787 & 0.537 \\
\hline Non-smoker & $237(90.5)$ & $238(90.5)$ & $230(87.5)$ & & \\
\hline Former smoker & $14(5.3)$ & $14(5.3)$ & $19(7.2)$ & & \\
\hline Current smoker & $11(4.2)$ & $11(4.2)$ & $14(5.3)$ & & \\
\hline Alcohol intake & & & & 0.022 & 0.227 \\
\hline Non-drinker & $50(27.0)$ & $62(23.0)$ & $60(18.0)$ & & \\
\hline Former drinker & $2(1.1)$ & $17(6.3)$ & $14(4.2)$ & & \\
\hline Current non-heavy drinker & $112(60.5)$ & $163(60.4)$ & $209(62.8)$ & & \\
\hline Current heavy drinker & $21(11.4)$ & $28(10.4)$ & $50(15.0)$ & & \\
\hline Regular exercise & $143(54.6)$ & $155(58.9)$ & $177(67.3)$ & 0.011 & 0.003 \\
\hline Sleep duration, hr/day & $6.9 \pm 1.3$ & $7.0 \pm 1.2$ & $7.0 \pm 1.2$ & 0.712 & 0.421 \\
\hline
\end{tabular}

Values are presented as mean \pm standard deviation, median (interquartile range), or number (\%). $P$ values were derived using the independent $t$ test, Wilcoxon rank sum test, or chi-square test.

TMA, thigh muscle area; Wt, weight; BMI, body mass index; SBP, systolic blood pressure; DBP, diastolic blood pressure; HDL-C, high-density lipoprotein cholesterol; LDL-C, low-density lipoprotein cholesterol; HbAlc, glycosylated hemoglobin; HOMA-IR, homeostasis model assessment of insulin resistance; IR, insulin resistance.

estimated muscle mass, instead of a measured muscle area.

Unlike previous studies, we measured TMA using QCT in a large general population and excluded the potential effect of an- tidiabetic treatment on the association between thigh muscle and IR, excluding the patients who were taking antidiabetic medications. We also tried to control for confounders not only 
Table 3. Characteristics in postmenopausal women $(n=1,476)$ according to TMA/Wt tertiles

\begin{tabular}{|c|c|c|c|c|c|}
\hline \multirow{2}{*}{ Variable } & \multicolumn{3}{|c|}{ TMA/Wt tertiles } & \multirow{2}{*}{$\begin{array}{c}P \text { for } \\
\text { difference }\end{array}$} & \multirow{2}{*}{$\begin{array}{l}P \text { for } \\
\text { trend }\end{array}$} \\
\hline & Lower $\left(<261.0 \mathrm{~cm}^{2} / \mathrm{kg}\right)$ & Middle $\left(261.0-292.4 \mathrm{~cm}^{2} / \mathrm{kg}\right)$ & Upper $\left(\geq 292.5 \mathrm{~cm}^{2} / \mathrm{kg}\right)$ & & \\
\hline Age, yr & $57.6 \pm 3.9$ & $57.0 \pm 3.9$ & $56.7 \pm 4.4$ & 0.001 & $<0.001$ \\
\hline BMI, kg/m² & $25.3 \pm 3.1$ & $23.5 \pm 2.4$ & $22.4 \pm 2.0$ & $<0.001$ & $<0.001$ \\
\hline Waist circumference, $\mathrm{cm}$ & $83.3 \pm 8.2$ & $79.3 \pm 6.8$ & $75.1 \pm 6.1$ & $<0.001$ & $<0.001$ \\
\hline Visceral fat area, $\mathrm{cm}^{2}$ & $115.90 \pm 40.40$ & $99.30 \pm 36.30$ & $79.90 \pm 30.90$ & $<0.001$ & $<0.001$ \\
\hline SBP, mm Hg & $119.9 \pm 14.9$ & $117.7 \pm 14.8$ & $114.8 \pm 13.2$ & $<0.001$ & $<0.001$ \\
\hline DBP, mm Hg & $76.1 \pm 9.0$ & $75.0 \pm 9.1$ & $73.2 \pm 8.3$ & $<0.001$ & $<0.001$ \\
\hline Total cholesterol, mg/dL & $209.1 \pm 36.0$ & $208.8 \pm 35.8$ & $203.8 \pm 33.2$ & 0.031 & 0.155 \\
\hline HDL-C, mg/dL & $59.3 \pm 13.6$ & $59.4 \pm 13.6$ & $62.2 \pm 15.0$ & 0.001 & 0.056 \\
\hline LDL-C, mg/dL & $128.0 \pm 32.0$ & $125.7 \pm 33.1$ & $121.2 \pm 31.3$ & 0.028 & 0.019 \\
\hline Triglycerides, mg/dL & $116.5(86.0-150.0)$ & $108.0(84.0-143.0)$ & $98.0(72.0-137.5)$ & $<0.001$ & 0.022 \\
\hline Fasting glucose, $\mathrm{mg} / \mathrm{dL}$ & $89.0(84.0-95.5)$ & $88.0(82.0-94.0)$ & $87.0(82.0-93.0)$ & $<0.001$ & $<0.001$ \\
\hline Fasting insulin, uIU/mL & $8.6(7.0-11.0)$ & $8.0(6.4-10.3)$ & $7.5(5.8-9.5)$ & $<0.001$ & $<0.001$ \\
\hline $\mathrm{HbA1c}, \%$ & $5.7(5.4-6.0)$ & $5.6(5.4-5.9)$ & $5.6(5.4-5.8)$ & 0.008 & $<0.001$ \\
\hline HOMA-IR & $1.89(1.50-2.59)$ & $1.74(1.35-2.29)$ & $1.58(1.25-2.12)$ & $<0.001$ & $<0.001$ \\
\hline Increased IR, \% & $160(32.5)$ & $118(24.0)$ & $91(18.5)$ & $<0.001$ & $<0.001$ \\
\hline C-reactive protein, $\mathrm{mg} / \mathrm{L}$ & $0.8(0.4-1.7)$ & $0.6(0.4-1.1)$ & $0.5(0.3-0.9)$ & $<0.001$ & 0.011 \\
\hline Hypertension & $159(32.3)$ & $131(26.6)$ & $105(21.3)$ & $<0.001$ & $<0.001$ \\
\hline Pre-diabetes mellitus & $238(48.4)$ & $199(40.5)$ & $214(43.5)$ & 0.041 & 0.123 \\
\hline Dyslipidemia & $254(51.6)$ & $233(47.4)$ & $195(39.6)$ & $<0.001$ & $<0.001$ \\
\hline Antihypertensive medication & $115(23.4)$ & $83(16.9)$ & $84(17.1)$ & 0.013 & 0.012 \\
\hline Lipid-lowering medication & $88(17.9)$ & $79(16.1)$ & $80(16.3)$ & 0.701 & 0.495 \\
\hline Smoking status & & & & 0.511 & 0.599 \\
\hline Non-smoker & $469(95.3)$ & $476(96.8)$ & $471(95.7)$ & & \\
\hline Former smoker & $15(3.1)$ & $12(2.4)$ & $11(2.2)$ & & \\
\hline Current smoker & $8(1.6)$ & $4(0.8)$ & $10(2.0)$ & & \\
\hline Alcohol intake & & & & 0.002 & 0.012 \\
\hline Non-drinker & $205(36.0)$ & $174(35.9)$ & $121(28.7)$ & & \\
\hline Former drinker & $17(3.0)$ & $19(3.9)$ & $9(2.1)$ & & \\
\hline Current non-heavy drinker & $317(55.7)$ & $272(56.1)$ & $251(59.5)$ & & \\
\hline Current heavy drinker & $30(2.3)$ & $20(4.1)$ & $41(9.7)$ & & \\
\hline Regular exercise & $326(66.3)$ & $332(67.5)$ & $355(72.2)$ & 0.109 & 0.046 \\
\hline Sleep duration, hr/day & $6.8 \pm 1.2$ & $6.8 \pm 1.2$ & $6.8 \pm 1.3$ & 0.802 & 0.507 \\
\hline
\end{tabular}

Values are presented as mean \pm standard deviation, median (interquartile range), or number (\%). $P$ values were derived using the independent $t$ test, Wilcoxon rank sum test, or chi-square test.

TMA, thigh muscle area; Wt, weight; BMI, body mass index; SBP, systolic blood pressure; DBP, diastolic blood pressure; HDL-C, high-density lipoprotein cholesterol; LDL-C, low-density lipoprotein cholesterol; HbAlc, glycosylated hemoglobin; HOMA-IR, homeostasis model assessment of insulin resistance; IR, insulin resistance.

by adjusting for risk factors, including central obesity, but also by stratifying by sex, menopausal status, and overall obesity status.

The underlying mechanisms of the association between low muscle mass and higher IR are not fully understood. One of the potential explanations is that muscle tissue is the primary tissue responsible for insulin-mediated glucose disposal; hence, 
Table 4. Association between TMA/Wt and increased IR in men and premenopausal and postmenopausal women

\begin{tabular}{|c|c|c|c|c|c|}
\hline \multirow{2}{*}{ Subgroup } & \multirow{2}{*}{ No. of participants } & \multirow{2}{*}{ No. of cases (\%) } & \multicolumn{3}{|c|}{ OR (95\% CI) } \\
\hline & & & Model 1 & Model 2 & Model 3 \\
\hline \multicolumn{6}{|l|}{ Men } \\
\hline Upper tertile & 421 & $54(12.8)$ & 1.00 & 1.00 & 1.00 \\
\hline Middle tertile & 421 & $92(21.9)$ & $1.98(1.37-2.87)$ & $1.67(1.14-2.45)$ & $1.05(0.70-1.58)$ \\
\hline Lower tertile & 421 & $170(40.4)$ & $4.79(3.38-6.79)$ & $3.87(2.70-5.55)$ & $1.63(1.10-2.45)$ \\
\hline Per $10 \mathrm{~cm}^{2} / \mathrm{kg}$ lower & 1,263 & $316(25.0)$ & $1.18(1.14-1.22)$ & $1.16(1.12-1.20)$ & $1.06(1.02-1.10)$ \\
\hline \multicolumn{6}{|l|}{ Premenopausal women } \\
\hline Upper tertile & 263 & $42(16.0)$ & 1.00 & 1.00 & 1.00 \\
\hline Middle tertile & 263 & $73(27.8)$ & $2.02(1.32-3.10)$ & $1.97(1.26-3.07)$ & $1.15(0.70-1.88)$ \\
\hline Lower tertile & 262 & $82(31.3)$ & $2.39(1.57-3.65)$ & $1.99(1.27-3.11)$ & $1.00(0.58-1.60)$ \\
\hline Per $10 \mathrm{~cm}^{2} / \mathrm{kg}$ lower & 788 & $197(25.0)$ & $1.12(1.08-1.17)$ & $1.10(1.05-1.15)$ & $1.01(0.95-1.06)$ \\
\hline \multicolumn{6}{|l|}{ Postmenopausal women } \\
\hline Upper tertile & 492 & $91(18.5)$ & 1.00 & 1.00 & 1.00 \\
\hline Middle tertile & 492 & $118(24.0)$ & $1.38(1.02-1.88)$ & $1.21(0.88-1.66)$ & $0.76(0.54-1.08)$ \\
\hline Lower tertile & 492 & $160(32.5)$ & $2.08(1.54-2.79)$ & $1.81(1.32-2.47)$ & $0.77(0.54-1.10)$ \\
\hline Per $10 \mathrm{~cm}^{2} / \mathrm{kg}$ lower & 1,476 & $369(25.0)$ & $1.08(1.05-1.11)$ & $1.07(1.04-1.10)$ & $0.97(0.93-1.01)$ \\
\hline
\end{tabular}

Model 1, adjusted for age; Model 2, adjusted for variables in Model 1 plus systolic blood pressure, triglyceride level, smoking, alcohol intake, regular exercise, and sleep duration; Model 3, adjusted for variables in Model 2 plus visceral fat.

TMA, thigh muscle area; Wt, weight; IR, insulin resistance; OR, odds ratio; CI, confidence interval.

Table 5. Association between TMA/Wt and increased IR according to BMI categorization in men

\begin{tabular}{|c|c|c|c|c|c|}
\hline \multirow{2}{*}{ BMI } & \multirow{2}{*}{ No. of participants } & \multirow{2}{*}{ No. (\%) of cases } & \multicolumn{3}{|c|}{ OR (95\% CI) } \\
\hline & & & Model 1 & Model 2 & Model 3 \\
\hline \multicolumn{6}{|l|}{$\mathrm{BMI}<25 \mathrm{~kg} / \mathrm{m}^{2}$} \\
\hline Upper tertile & 227 & $12(5.3)$ & 1.00 & 1.00 & 1.00 \\
\hline Middle tertile & 228 & $32(14.0)$ & $2.93(1.46-5.85)$ & $2.58(1.26-5.28)$ & $1.84(0.88-3.84)$ \\
\hline Lower tertile & 227 & $36(15.9)$ & $3.38(1.70-6.70)$ & $3.15(1.57-6.34)$ & $1.59(0.75-3.40)$ \\
\hline Per $10 \mathrm{~cm}^{2} / \mathrm{kg}$ lower & 682 & $80(11.7)$ & $1.10(1.04-1.17)$ & $1.10(1.03-1.16)$ & $1.02(0.95-1.09)$ \\
\hline \multicolumn{6}{|l|}{$\mathrm{BMI} \geq 25 \mathrm{~kg} / \mathrm{m}^{2}$} \\
\hline Upper tertile & 194 & $52(26.8)$ & 1.00 & 1.00 & 1.00 \\
\hline Middle tertile & 194 & $71(36.6)$ & $1.59(1.03-2.45)$ & $1.54(0.98-2.43)$ & $1.08(0.66-1.74)$ \\
\hline Lower tertile & 193 & $113(58.6)$ & $3.89(2.53-5.97)$ & $3.73(2.39-5.85)$ & $2.31(1.42-3.74)$ \\
\hline Per $10 \mathrm{~cm}^{2} / \mathrm{kg}$ lower & 581 & $236(40.6)$ & $1.16(1.11-1.21)$ & $1.15(1.10-1.20)$ & $1.09(1.03-1.15)$ \\
\hline
\end{tabular}

Model 1, adjusted for age; Model 2: adjusted for variables in Model 1 plus systolic blood pressure, triglyceride level, smoking, alcohol intake, regular exercise, and sleep duration; Model 3, adjusted for variables in Model 2 plus visceral fat.

TMA, thigh muscle area; Wt, weight; IR, insulin resistance; BMI, body mass index; OR, odds ratio; CI, confidence interval.

a low muscle mass could cause a decreased insulin-mediated glucose disposal, followed by higher IR. Another explanation could be in lifestyle factors, including smoking, alcohol intake, and physical activity. In the present study, after adjusting for these lifestyle factors, ORs were slightly weakened, but still remained significant in men. This suggested that lifestyle factors might partially, but not fully, explain the association between low muscle mass and higher IR. In the current study, according 
to stratification analyses by BMI categories, a significant inverse association was only observed in men with higher BMIs. Interestingly, while age-related muscle loss has been shown to be due to a reduction in muscle fibers and fiber atrophy [30], participants with obesity [31-33], particularly men [34], also have increased ceramides in their muscle fibers, as well as fewer muscle fibers, which plays an important role in IR.

In women, regardless of menopausal status, the significant association disappeared after adjusting for the visceral fat area. This finding could suggest that the differences in body composition between men and women may modify the relationship between muscle and IR. First, age-related decreases in skeletal muscle mass and muscle strength are inevitable in both men and women; however, they are more prominent in men than in women [12]. This could be affected by the sex-specific effects of sex hormones. In men, testosterone levels generally control changes in skeletal muscle mass, and in women, there is much less of an absolute decline in testosterone levels with aging than that in men. Thus, women may not experience the effects of testosterone decline on skeletal muscle mass. Additionally, female sex hormones, especially estrogen, have been found to provide protective effects on adipocyte inflammation, oxidative stress, and IR in an animal study [35]. Second, fat distributions are different between men and women. Adipose tissue primarily accumulates around the trunk and abdomen in men, but around the hips and thighs in women [36,37]. According to previous studies, although the absolute amount of visceral fat area is higher in men than in women, the risk of visceral fat area on cardiometabolic abnormalities is higher in women than in men [38]. A possible reason for this is that the thigh muscle might have a relatively smaller impact on the IR in women than in men [39], due to the preexisting risk of fat on metabolic imbalance [40]. In this regard, women would likely be less affected by muscle declines than men.

After stratification according to menopausal status, lower thigh muscle tended to be associated with higher IR in premenopausal women, whereas higher thigh muscle was tended to be associated with higher IR in postmenopausal women. Nevertheless, both of these trends were not significant. Instead of thigh muscle mass, age and visceral fat area were significantly associated with increased IR in premenopausal women, while blood pressure, triglycerides, and visceral fat area were significantly associated with increased IR in postmenopausal women (Supplementary Table 3). Several previous studies [4143] have reported that postmenopausal women have greater abdominal fat, higher blood pressure, higher lipid profiles than premenopausal women even after adjustment for age, and these risk factors could have great harmful impact on higher IR than protective effect of greater muscle mass. Further studies are needed to confirm which ways associations differ between premenopausal and postmenopausal women, as well as between men and women.

The current study had a few limitations. First, TMA was measured using QCT, but more detailed characteristics such as thigh intramuscular or intermuscular fat content were not measured. Previous research has indicated that there are sex differences in mid-thigh composition, such as muscle density, which is a marker of muscle fat infiltration $[44,45]$. Although fat infiltration in a muscle accounts for a relatively small portion of the muscle, not considering muscle fat infiltration may not represent the muscles of women well. Second, we used HOMA-IR as a surrogate marker for IR. However, it is frequently used for assessing IR in large population-based studies, because it takes minimal time, is not invasive, and shows excellent predictability for IR [46]. Third, both the thigh muscle and IR were measured only once; therefore, we cannot exclude the possibility of random measurement errors that might have weakened the observed association. Fourth, we might not have completely accounted for all residual confounders. Finally, this study was cross-sectional in nature; therefore, a causal association between low thigh muscle mass and higher IR is uncertain.

In conclusion, we found an association between lower thigh muscle mass and higher IR in men, particularly those with higher BMIs, but not in those with lower BMIs, even after adjusting for traditional risk factors. Our findings suggest that the maintenance of both an appropriate BMI and thigh muscle is important for normal IR in middle-aged Korean men.

\section{SUPPLEMENTARY MATERIALS}

Supplementary materials related to this article can be found online at https://doi.org/10.4093/dmj.2019.0110.

\section{CONFLICTS OF INTEREST}

No potential conflict of interest relevant to this article was reported. 


\section{AUTHOR CONTRIBUTIONS}

Conception or design: J.E.H., H.C.K.

Acquisition, analysis, or interpretation of data: J.E.H., J.S.S., H.C.K.

Drafting the work or revising: J.E.H., H.L., H.C.K.

Final approval of the manuscript: J.E.H., J.S.S., H.L., H.C.K.

\section{ORCID}

Ji Eun Heo https://orcid.org/0000-0001-6449-5856

Hyeon Chang Kim https://orcid.org/0000-0001-7867-1240

\section{ACKNOWLEDGMENTS}

This work was supported by the Korea Health Technology R\&D Project (grant number: HI13C0715) funded by the Ministry of Health and Welfare of the Republic of Korea.

\section{REFERENCES}

1. Eastwood SV, Tillin T, Wright A, Mayet J, Godsland I, Forouhi NG, Whincup P, Hughes AD, Chaturvedi N. Thigh fat and muscle each contribute to excess cardiometabolic risk in South Asians, independent of visceral adipose tissue. Obesity (Silver Spring) 2014;22:2071-9.

2. Moon SS. Low skeletal muscle mass is associated with insulin resistance, diabetes, and metabolic syndrome in the Korean population: the Korea National Health and Nutrition Examination Survey (KNHANES) 2009-2010. Endocr J 2014;61:61-70.

3. Heo JE, Shim JS, Song BM, Bae HY, Lee HJ, Lee E, Kim HC. Association between appendicular skeletal muscle mass and depressive symptoms: review of the cardiovascular and metabolic diseases etiology research center cohort. J Affect Disord 2018;238:8-15.

4. Lee SW, Youm Y, Lee WJ, Choi W, Chu SH, Park YR, Kim HC. Appendicular skeletal muscle mass and insulin resistance in an elderly Korean population: the Korean social life, health and aging project-health examination cohort. Diabetes Metab J 2015;39:37-45.

5. Hirasawa Y, Matsuki R, Ebisu T, Kurose T, Hamamoto Y, Seino Y. Evaluation of skeletal muscle mass indices, assessed by bioelectrical impedance, as indicators of insulin resistance in patients with type 2 diabetes. J Phys Ther Sci 2019;31:190-4.

6. Wells CE, Polkey MI, Baker EH. Insulin resistance is associated with skeletal muscle weakness in COPD. Respirology 2016;21: 689-96.

7. Wang HL, Ding TT, Lu S, Xu Y, Tian J, Hu WF, Zhang JY. Muscle mass loss and intermuscular lipid accumulation were associated with insulin resistance in patients receiving hemodialysis. Chin Med J (Engl) 2013;126:4612-7.

8. Lee J, Hong YP, Shin HJ, Lee W. Associations of sarcopenia and sarcopenic obesity with metabolic syndrome considering both muscle mass and muscle strength. J Prev Med Public Health 2016;49:35-44

9. Poehlman ET, Dvorak RV, DeNino WF, Brochu M, Ades PA. Effects of resistance training and endurance training on insulin sensitivity in nonobese, young women: a controlled randomized trial. J Clin Endocrinol Metab 2000;85:2463-8.

10. Grontved A, Ried-Larsen M, Ekelund U, Froberg K, Brage S, Andersen LB. Independent and combined association of muscle strength and cardiorespiratory fitness in youth with insulin resistance and $\beta$-cell function in young adulthood: the European Youth Heart Study. Diabetes Care 2013;36:2575-81.

11. Srikanthan P, Karlamangla AS. Relative muscle mass is inversely associated with insulin resistance and prediabetes: findings from the third National Health and Nutrition Examination Survey. J Clin Endocrinol Metab 2011;96:2898-903.

12. Kim KM, Jang HC, Lim S. Differences among skeletal muscle mass indices derived from height-, weight-, and body mass index-adjusted models in assessing sarcopenia. Korean J Intern Med 2016;31:643-50.

13. Dmitruk A, Czeczelewski J, Czeczelewska E, Golach J, Parnicka U. Body composition and fatty tissue distribution in women with various menstrual status. Rocz Panstw Zakl Hig 2018;69: 95-101.

14. Moon HU, Ha KH, Han SJ, Kim HJ, Kim DJ. The association of adiponectin and visceral fat with insulin resistance and $\beta$-cell dysfunction. J Korean Med Sci 2018;34:e7.

15. Cheng YH, Tsao YC, Tzeng IS, Chuang HH, Li WC, Tung TH, Chen JY. Body mass index and waist circumference are better predictors of insulin resistance than total body fat percentage in middle-aged and elderly Taiwanese. Medicine (Baltimore) 2017;96:e8126.

16. Peplies J, Bornhorst C, Gunther K, Fraterman A, Russo P, Veidebaum T, Tornaritis M, De Henauw S, Marild S, Molnar D, Moreno LA, Ahrens W; IDEFICS consortium. Longitudinal associations of lifestyle factors and weight status with insulin resistance (HOMA-IR) in preadolescent children: the large prospective cohort study IDEFICS. Int J Behav Nutr Phys Act 
2016;13:97.

17. Shim JS, Song BM, Lee JH, Lee SW, Park JH, Choi DP, Lee MH, Ha KH, Kim DJ, Park S, Lee WW, Kim HC. Cardiovascular and Metabolic Diseases Etiology Research Center (CMERC) cohort: study protocol and results of the first 3 years of enrollment. Epidemiol Health 2017;39:e2017016.

18. Park JT, Kim BG, Jhun HJ. Alcohol consumption and the CAGE questionnaire in Korean adults: results from the Second Korea National Health and Nutrition Examination Survey. J Korean Med Sci 2008;23:199-206.

19. Matthews DR, Hosker JP, Rudenski AS, Naylor BA, Treacher DF, Turner RC. Homeostasis model assessment: insulin resistance and beta-cell function from fasting plasma glucose and insulin concentrations in man. Diabetologia 1985;28:412-9.

20. Yun KJ, Han K, Kim MK, Park YM, Baek KH, Song KH, Kwon HS. Insulin resistance distribution and cut-off value in Koreans from the 2008-2010 Korean National Health and Nutrition Examination Survey. PLoS One 2016;11:e0154593.

21. American Diabetes Association. Diagnosis and classification of diabetes mellitus. Diabetes Care 2012;35 Suppl 1(Suppl 1):S6471.

22. Ochi M, Tabara Y, Kido T, Uetani E, Ochi N, Igase M, Miki T, Kohara K. Quadriceps sarcopenia and visceral obesity are risk factors for postural instability in the middle-aged to elderly population. Geriatr Gerontol Int 2010;10:233-43.

23. Huang L, Tepaamorndech S, Kirschke CP, Newman JW, Keyes WR, Pedersen TL, Dumnil J. Aberrant fatty acid metabolism in skeletal muscle contributes to insulin resistance in zinc transporter 7 (znt7)-knockout mice. J Biol Chem 2018;293:7549-63.

24. Srikanthan P, Hevener AL, Karlamangla AS. Sarcopenia exacerbates obesity-associated insulin resistance and dysglycemia: findings from the National Health and Nutrition Examination Survey III. PLoS One 2010;5:e10805.

25. Kim J. Gender difference in association between appendicular skeletal muscle mass and cardiometabolic abnormalities in normal-weight and obese adults: Korea National Health and Nutrition Examination Survey (KNHANES) IV-3 and V-1. Asia Pac J Public Health 2015;27:NP468-75.

26. Han SJ, Boyko EJ, Kim SK, Fujimoto WY, Kahn SE, Leonetti DL. Association of thigh muscle mass with insulin resistance and incident type 2 diabetes mellitus in Japanese Americans. Diabetes Metab J 2018;42:488-95.

27. Aleman-Mateo H, Lopez Teros MT, Ramirez FA, AstiazaranGarcia H. Association between insulin resistance and low relative appendicular skeletal muscle mass: evidence from a cohort study in community-dwelling older men and women participants. J Gerontol A Biol Sci Med Sci 2014;69:871-7.

28. Tonks KT, Coster AC, Christopher MJ, Chaudhuri R, Xu A, Gagnon-Bartsch J, Chisholm DJ, James DE, Meikle PJ, Greenfield JR, Samocha-Bonet D. Skeletal muscle and plasma lipidomic signatures of insulin resistance and overweight/obesity in humans. Obesity (Silver Spring) 2016;24:908-16.

29. Fu X, Zhu F, Zhao X, Ma X, Zhu S. Central fat accumulation associated with metabolic risks beyond total fat in normal BMI Chinese adults. Ann Nutr Metab 2014;64:93-100.

30. Gueugneau M, Coudy-Gandilhon C, Theron L, Meunier B, Barboiron C, Combaret L, Taillandier D, Polge C, Attaix D, Picard B, Verney J, Roche F, Feasson L, Barthelemy JC, Bechet D. Skeletal muscle lipid content and oxidative activity in relation to muscle fiber type in aging and metabolic syndrome. J Gerontol A Biol Sci Med Sci 2015;70:566-76.

31. Adams JM 2nd, Pratipanawatr T, Berria R, Wang E, DeFronzo RA, Sullards MC, Mandarino LJ. Ceramide content is increased in skeletal muscle from obese insulin-resistant humans. Diabetes 2004;53:25-31.

32. Boden G. Ceramide: a contributor to insulin resistance or an innocent bystander? Diabetologia 2008;51:1095-6.

33. Coen PM, Dube JJ, Amati F, Stefanovic-Racic M, Ferrell RE, Toledo FG, Goodpaster BH. Insulin resistance is associated with higher intramyocellular triglycerides in type I but not type II myocytes concomitant with higher ceramide content. Diabetes 2010;59:80-8.

34. Haugaard SB, Vaag A, Hoy CE, Madsbad S. Sex and muscle structural lipids in obese subjects: an impact on insulin action? Eur J Clin Invest 2008;38:494-501.

35. Stubbins RE, Najjar K, Holcomb VB, Hong J, Nunez NP. Oestrogen alters adipocyte biology and protects female mice from adipocyte inflammation and insulin resistance. Diabetes Obes Metab 2012;14:58-66.

36. Bredella MA. Sex differences in body composition. Adv Exp Med Biol 2017;1043:9-27.

37. Wu CH, Yao WJ, Lu FH, Yang YC, Wu JS, Chang CJ. Sex differences of body fat distribution and cardiovascular dysmetabolic factors in old age. Age Ageing 2001;30:331-6.

38. Schorr M, Dichtel LE, Gerweck AV, Valera RD, Torriani M, Miller KK, Bredella MA. Sex differences in body composition and association with cardiometabolic risk. Biol Sex Differ 2018; 9:28.

39. Larsen BA, Wassel CL, Kritchevsky SB, Strotmeyer ES, Criqui MH, Kanaya AM, Fried LF, Schwartz AV, Harris TB, Ix JH; 
Health ABC Study. Association of muscle mass, area, and strength with incident diabetes in older adults: the Health $A B C$ Study. J Clin Endocrinol Metab 2016;101:1847-55.

40. Brochu M, Starling RD, Tchernof A, Matthews DE, Garcia-Rubi E, Poehlman ET. Visceral adipose tissue is an independent correlate of glucose disposal in older obese postmenopausal women. J Clin Endocrinol Metab 2000;85:2378-84.

41. Toth MJ, Tchernof A, Sites CK, Poehlman ET. Menopause-related changes in body fat distribution. Ann N Y Acad Sci 2000; 904:502-6.

42. Zanchetti A, Facchetti R, Cesana GC, Modena MG, Pirrelli A, Sega R; SIMONA participants. Menopause-related blood pressure increase and its relationship to age and body mass index: the SIMONA epidemiological study. J Hypertens 2005;23: 2269-76.

43. Wang N, Qin MZ, Cui J. Lipid profile comparison between pre- and post-menopausal women. Zhonghua Xin Xue Guan Bing Za Zhi 2016;44:799-804.

44. Kasai T, Ishiguro N, Matsui Y, Harada A, Takemura M, Yuki A, Kato Y, Otsuka R, Ando F, Shimokata H. Sex- and age-related differences in mid-thigh composition and muscle quality determined by computed tomography in middle-aged and elderly Japanese. Geriatr Gerontol Int 2015;15:700-6.

45. Visser M, Goodpaster BH, Kritchevsky SB, Newman AB, Nevitt M, Rubin SM, Simonsick EM, Harris TB. Muscle mass, muscle strength, and muscle fat infiltration as predictors of incident mobility limitations in well-functioning older persons. J Gerontol A Biol Sci Med Sci 2005;60:324-33.

46. Ikeda Y, Suehiro T, Nakamura T, Kumon Y, Hashimoto K. Clinical significance of the insulin resistance index as assessed by homeostasis model assessment. Endocr J 2001;48:81-6. 